\title{
Global Existence of Solution to Initial Boundary Value Problem for Bipolar Navier-Stokes-Poisson System
}

\author{
Jian Liu ${ }^{1}$ and Haidong Liu ${ }^{2}$ \\ ${ }^{1}$ College of Teacher Education, Quzhou University, Quzhou 324000, China \\ ${ }^{2}$ College of Mathematics, Physics and Information Engineering, Jiaxing University, Jiaxing 314001, China \\ Correspondence should be addressed to Haidong Liu; liuhaidong@mail.zjxu.edu.cn
}

Received 2 June 2014; Accepted 12 August 2014; Published 28 August 2014

Academic Editor: Xiaohong Qin

Copyright (c) $2014 \mathrm{~J}$. Liu and H. Liu. This is an open access article distributed under the Creative Commons Attribution License, which permits unrestricted use, distribution, and reproduction in any medium, provided the original work is properly cited.

\begin{abstract}
This paper concerns initial boundary value problem for 3-dimensional compressible bipolar Navier-Stokes-Poisson equations with density-dependent viscosities. When the initial data is large, discontinuous, and spherically symmetric, we prove the global existence of the weak solution.
\end{abstract}

\section{Introduction}

Bipolar Navier-Stokes-Poisson (BNSP) has been used to simulate the transport of charged particles under the influence of electrostatic force governed by the self-consistent Poisson equation. In this paper, we consider the initial boundary value problem (IBVP) for 3-dimensional isentropic compressible BNSP with density-dependent viscosities:

$$
\begin{gathered}
\rho_{t}+\operatorname{div}(\rho \mathbf{U})=0, \\
(\rho \mathbf{U})_{t}+\operatorname{div}(\rho \mathbf{U} \otimes \mathbf{U})+\nabla P(\rho) \\
=\operatorname{div}(h(\rho) \mathbb{D}(\mathbf{U}))+\nabla(g(\rho) \operatorname{div} \mathbf{U})+\rho \nabla \Phi, \\
n_{t}+\operatorname{div}(n \mathbf{V})=0, \\
(n \mathbf{V})_{t}+\operatorname{div}(n \mathbf{V} \otimes \mathbf{V})+\nabla P(n) \\
=\operatorname{div}(h(n) \mathbb{D}(\mathbf{V}))+\nabla(g(n) \operatorname{div} \mathbf{V})-n \nabla \Phi, \\
\Delta \Phi=\rho-n,
\end{gathered}
$$

where the unknown functions are the charges densities $\rho(\mathbf{x}, t), n(\mathbf{x}, t)$, the velocities $\mathbf{U}(\mathbf{x}, t), \mathbf{V}(\mathbf{x}, t)$, the pressure functions $P(\rho)=\rho^{\gamma}, P(n)=n^{\gamma}(\gamma>1)$, and the electrostatic potential $\Phi(\mathbf{x}, t)$. In (1), the strain tensors $\mathbb{D}(\mathbf{U})$ and $\mathbb{D}(\mathbf{V})$ are defined by $\mathbb{D}(\mathbf{U})=(1 / 2)\left(\nabla \mathbf{U}+\nabla \mathbf{U}^{T}\right), \mathbb{D}(\mathbf{V})=(1 / 2)$ $\left(\nabla \mathbf{V}+\nabla \mathbf{V}^{T}\right)$, and the Lamé viscosity coefficients satisfying $h(\rho) \geq 0, h(\rho)+N g(\rho) \geq 0, h(n) \geq 0, h(n)+N g(n) \geq 0$.

There have been extensive studies on the global existence and asymptotic behavior of weak solution to the unipolar Navier-Stokes-Poisson system (NSP). The global existence of weak solution to NSP with general initial data was proved in $[1,2]$. The quasineutral and some related asymptotic limits were studied in [3-5]. In the case when the Poisson equation describes the self-gravitational force for stellar gases, the global existence of weak solution and asymptotic behavior were also investigated together with the stability analysis; refer to $[6,7]$ and the references therein. In addition, Hao and Li $[8]$ proved the global well-posedness of NSP in the Besov space. Li et al. in [9] proved the global existence and the optimal time convergence rates of the classical solution.

For bipolar Navier-Stokes-Poisson system, there are also abundant results on the existence and asymptotic behavior of the global solution. Li et al. [10] proved optimal $L^{2}$ time convergence rate for the global classical solution for a small initial perturbation of the constant equilibrium state. The optimal time decay rate of global strong solution was established in [11, 12]. Liu and Lian in [13] proved global existence of weak solution to free boundary value problem. Liu et al. [14] established global existence and asymptotic behavior of weak solution to initial boundary value problem in one-dimensional case. Lin et al. [15] studied the global existence and uniqueness of the strong solution in hybrid 
Besov spaces with the initial data close to an equilibrium state. As a continuation of the study in this direction, in this paper, we will deal with the initial boundary value problem for BNSP.

The rest of this paper is as follows. In Section 2, we state the main results of this paper. In Section 3, we give the entropy estimates and the pointwise bounds of the density of the smooth approximate solution. In Section 4, we prove the global existence of weak solution.

\section{Main Results}

For the sake of simplicity, the viscosity terms are assumed to satisfy $h(\rho)=\rho, g(\rho)=0, h(n)=n$, and $g(n)=0$, and the strain tensors are given by $\mathbb{D}(\mathbf{U})=\nabla \mathbf{U}, \mathbb{D}(\mathbf{V})=\nabla \mathbf{V}$. Then (1) is reduced to

$$
\begin{gathered}
\rho_{t}+\operatorname{div}(\rho \mathbf{U})=0, \\
(\rho \mathbf{U})_{t}+\operatorname{div}(\rho \mathbf{U} \otimes \mathbf{U})+\nabla \rho^{\gamma}=\rho \nabla \Phi+\nabla \cdot(\rho \nabla \mathbf{U}), \\
n_{t}+\operatorname{div}(n \mathbf{V})=0, \\
(n \mathbf{V})_{t}+\operatorname{div}(n \mathbf{V} \otimes \mathbf{V})+\nabla n^{\gamma}=-n \nabla \Phi+\nabla \cdot(n \nabla \mathbf{V}), \\
\Delta \Phi=\rho-n,
\end{gathered}
$$

for $(\mathbf{x}, t) \in \Omega \times[0, T]$ with $T>0$ and $\Omega$ being the unit ball in $R^{3}$.

The boundary condition is taken as

$$
\begin{array}{r}
\mathbf{m}_{1}(\mathbf{x}, t)=\rho(\mathbf{x}, t) \mathbf{U}(\mathbf{x}, t)=0, \\
\mathbf{m}_{2}(\mathbf{x}, t)=n(\mathbf{x}, t) \mathbf{V}(\mathbf{x}, t)=0, \\
\nabla \Phi \cdot \nu=0, \\
\mathbf{x} \in \partial \Omega,
\end{array}
$$

where $v$ is outward pointing unit normal vector of $\Omega$.

The initial data is

$$
\begin{gathered}
(\rho, \mathbf{U}, n, \mathbf{V}, \nabla \Phi)(\mathbf{x}, 0)=\left(\rho_{0}, \mathbf{U}_{0}, n_{0}, \mathbf{V}_{0}, \nabla \Phi_{0}\right)(\mathbf{x}), \quad \mathbf{x} \in \Omega, \\
\mathbf{m}_{10}(\mathbf{x}, 0)=\rho_{0}(\mathbf{x}) \mathbf{U}_{0}(\mathbf{x}), \\
\mathbf{m}_{20}(\mathbf{x}, 0)=n_{0}(\mathbf{x}) \mathbf{V}_{0}(\mathbf{x}), \\
\mathbf{x} \in \Omega .
\end{gathered}
$$

Definition 1. $(\rho, \mathbf{U}, n, \mathbf{V}, \nabla \Phi)$ is said to be a weak solution to the initial boundary value problem (2)-(4) on $\Omega \times[0, T]$, provided that

$$
\begin{gathered}
0 \leq \rho, n \in L^{\infty}\left(0, T ; L^{1}(\Omega) \cap L^{\gamma}(\Omega)\right), \\
\rho \nabla \mathbf{U}, n \nabla \mathbf{V} \in L^{2}\left(0, T ; W^{1,-1}(\Omega)\right), \\
\sqrt{\rho}, \sqrt{n} \in L^{\infty}\left(0, T ; H^{1}(\Omega)\right) \\
\sqrt{\rho} \mathbf{U}, \sqrt{n} \mathbf{V} \in L^{\infty}\left(0, T ; L^{2}(\Omega)\right), \\
\nabla \Phi \in L^{\infty}\left(0, T ; W^{1,1}(\Omega)\right)
\end{gathered}
$$

and the equations are satisfied in the sense of distributions. Namely, it holds for any $t_{2}>t_{1} \geq 0$ and $\phi \in C^{1}(\bar{\Omega} \times[0, T])$ that

$$
\begin{aligned}
& \left.\int_{\Omega} \rho \phi \mathrm{d} \mathbf{x}\right|_{t=t_{1}} ^{t_{2}}=\int_{t_{1}}^{t_{2}} \int_{\Omega}\left(\rho \phi_{t}+\rho \mathbf{U} \cdot \nabla \phi\right) \mathrm{d} \mathbf{x} \mathrm{d} t \\
& \left.\int_{\Omega} n \phi \mathrm{d} \mathbf{x}\right|_{t=t_{1}} ^{t_{2}}=\int_{t_{1}}^{t_{2}} \int_{\Omega}\left(n \phi_{t}+n \mathbf{V} \cdot \nabla \phi\right) \mathrm{d} \mathbf{x} \mathrm{d} t,
\end{aligned}
$$

and for $\psi=\left(\psi^{1}, \psi^{2}, \psi^{3}\right) \in C^{1}(\bar{\Omega} \times[0, T])$ satisfying $\psi(\mathbf{x}, t)=0$ on $\partial \Omega$ and $\psi(\mathbf{x}, T)=0$ that

$$
\begin{aligned}
& \int_{\Omega} \mathbf{m}_{10} \cdot \psi(\mathbf{x}, 0) \mathrm{d} \mathbf{x} \\
& +\int_{0}^{T} \int_{\Omega}\left[\sqrt{\rho}(\sqrt{\rho} \mathbf{U}) \cdot \partial_{t} \psi+\sqrt{\rho} \mathbf{U} \otimes \sqrt{\rho} \mathbf{U}: \nabla \psi\right] \mathrm{d} \mathbf{x} \mathrm{d} t \\
& +\int_{0}^{T} \int_{\Omega} \rho^{\gamma} \operatorname{div} \psi \mathrm{d} \mathbf{x} \mathrm{d} t+\int_{0}^{T} \int_{\Omega} \rho \psi \cdot \nabla \Phi \mathrm{d} \mathbf{x} \mathrm{d} t \\
& -\int_{0}^{T} \int_{\Omega} \rho \nabla \mathbf{U}: \nabla \psi \mathrm{d} \mathbf{x} \mathrm{d} t=0
\end{aligned}
$$

$$
\begin{aligned}
& \int_{\Omega} \mathbf{m}_{20} \cdot \psi(\mathbf{x}, 0) \mathrm{d} \mathbf{x} \\
& \quad+\int_{0}^{T} \int_{\Omega}\left[\sqrt{n}(\sqrt{n} \mathbf{V}) \cdot \partial_{t} \psi+\sqrt{n} \mathbf{V} \otimes \sqrt{n} \mathbf{V}: \nabla \psi\right] \mathrm{d} \mathbf{x} \mathrm{d} t \\
& \quad+\int_{0}^{T} \int_{\Omega} n^{\gamma} \operatorname{div} \psi \mathrm{d} \mathbf{x} \mathrm{d} t-\int_{0}^{T} \int_{\Omega} n \psi \cdot \nabla \Phi \mathrm{d} \mathbf{x} \mathrm{d} t \\
& \quad-\int_{0}^{T} \int_{\Omega} n \nabla \mathbf{V}: \nabla \psi \mathrm{d} \mathbf{x} \mathrm{d} t=0 .
\end{aligned}
$$

Before stating the main result, we make the following assumptions on the initial data (4):

$$
\begin{gathered}
\rho_{0}=\rho_{0}(|\mathbf{x}|), \quad \mathbf{U}_{0}=u_{0}(|\mathbf{x}|) \frac{\mathbf{x}}{r}, \quad n_{0}=n_{0}(|\mathbf{x}|), \\
\mathbf{V}_{0}=v_{0}(|\mathbf{x}|) \frac{\mathbf{x}}{r}, \\
\rho_{0} \geq 0 \quad \text { a.e. in } \Omega, \quad \rho_{0} \in W^{1,4}(\Omega), \\
n_{0} \geq 0 \quad \text { a.e. in } \Omega, \quad n_{0} \in W^{1,4}(\Omega), \\
\mathbf{m}_{10}=0, \quad \text { a.e. on }\left\{\mathbf{x} \in \Omega \mid \rho_{0}(\mathbf{x})=0\right\}, \\
\mathbf{m}_{10}^{4} \in L^{1}(\Omega), \quad \frac{\mathbf{m}_{10}^{2+\eta}}{\rho_{0}^{1+\eta}} \in L^{1}(\Omega),
\end{gathered}
$$




$$
\begin{gathered}
\mathbf{m}_{20}=0, \quad \text { a.e. on }\left\{\mathbf{x} \in \Omega \mid n_{0}(\mathbf{x})=0\right\}, \\
\mathbf{m}_{20}^{4} \in L^{1}(\Omega), \quad \frac{\mathbf{m}_{20}^{2+\eta}}{n_{0}^{1+\eta}} \in L^{1}(\Omega), \\
\nabla \sqrt{\rho_{0}} \in L^{2}(\Omega), \quad \nabla \sqrt{n_{0}} \in L^{2}(\Omega), \quad \nabla \Phi_{0} \in L^{2}(\Omega),
\end{gathered}
$$

where $\eta>0$ is small enough. It follows from (9) that

$$
\begin{aligned}
& \rho_{0} \in L^{\infty}(\Omega) ; \quad \rho_{0} \mathbf{U}_{0}^{2+\eta} \in L^{1}(\Omega) ; \quad \rho_{0} \mathbf{U}_{0}^{2} \in L^{1}(\Omega), \\
& n_{0} \in L^{\infty}(\Omega) ; \quad n_{0} \mathbf{V}_{0}^{2+\eta} \in L^{1}(\Omega) ; \quad n_{0} \mathbf{V}_{0}^{2} \in L^{1}(\Omega) \text {. }
\end{aligned}
$$

Then, we have the following results for global weak solution.

Theorem 2. Let $1<\gamma<3$. If the initial data satisfies (9), then the initial boundary value problem (2)-(4) has a global spherically symmetric weak solution

$$
\begin{array}{r}
(\rho, \mathbf{U}, n, \mathbf{V}, \nabla \Phi)(\mathbf{x}, t) \\
=\left(\rho(r, t), u(r, t) \frac{\mathbf{x}}{r}, n(r, t), v(r, t) \frac{\mathbf{x}}{r}, \nabla \Phi(r, t)\right), \\
r=|\mathbf{x}|,
\end{array}
$$

which satisfies, for all $T>0$,

$$
\begin{aligned}
& \rho(\mathbf{x}, t) \in C\left([0, T] ; L^{3 / 2}(\Omega)\right), \\
& n(\mathbf{x}, t) \in C\left([0, T] ; L^{3 / 2}(\Omega)\right), \\
& \sqrt{\rho} \mathbf{U} \in L^{\infty}\left([0, T] ; L^{2}(\Omega)\right), \\
& \sqrt{n} \mathbf{V} \in L^{\infty}\left([0, T] ; L^{2}(\Omega)\right), \\
& \int_{\Omega} \rho(\mathbf{x}, t) \mathrm{d} \mathbf{x}=\int_{\Omega} \rho_{0}(\mathbf{x}) \mathrm{d} \mathbf{x}, \\
& \int_{\Omega} n(\mathbf{x}, t) \mathrm{d} \mathbf{x}=\int_{\Omega} n_{0}(\mathbf{x}) \mathrm{d} \mathbf{x}, \\
& \nabla \Phi \in L^{\infty}\left([0, T] ; W^{1,3 / 2}(\Omega)\right) .
\end{aligned}
$$

Moreover,

$$
\begin{array}{r}
\sup _{t \in[0, T]} \int_{\Omega}\left(\frac{1}{2} \rho|\mathbf{U}|^{2}+\frac{1}{2} n|\mathbf{V}|^{2}+\frac{1}{\gamma-1} \rho^{\gamma}\right. \\
\left.+\frac{1}{\gamma-1} n^{\gamma}+\frac{1}{2}|\nabla \Phi|^{2}\right) \mathrm{d} \mathbf{x} \leq C, \\
\sup _{t \in[0, T]} \int_{\Omega}\left(|\nabla \sqrt{\rho}|^{2}+|\nabla \sqrt{n}|^{2}\right) \mathrm{d} \mathbf{x} \leq C,
\end{array}
$$

where $C>0$ is a constant.

\section{Approximate Solutions and Their Estimates}

The key point of the proof of Theorem 2 is to construct smooth approximate solution satisfying the a priori estimates required in the $L^{1}$ stability analysis. The crucial issue is to obtain lower and upper bounds of the density. To this end, we study the following approximate system of (2):

$$
\rho_{t}+\operatorname{div}(\rho \mathbf{U})=0
$$

$$
\begin{gathered}
(\rho \mathbf{U})_{t}+\operatorname{div}(\rho \mathbf{U} \otimes \mathbf{U})-\operatorname{div}\left(\left(\rho+\varepsilon \rho^{3 / 4}\right) \nabla \mathbf{U}\right) \\
+\nabla\left(\frac{\varepsilon}{4} \rho^{3 / 4} \operatorname{div} \mathbf{U}\right)+\nabla \rho^{\gamma}=\rho \nabla \Phi, \\
n_{t}+\operatorname{div}(n \mathbf{V})=0, \\
(n \mathbf{V})_{t}+\operatorname{div}(n \mathbf{V} \otimes \mathbf{V})-\operatorname{div}\left(\left(n+\varepsilon n^{3 / 4}\right) \nabla \mathbf{V}\right) \\
+\nabla\left(\frac{\varepsilon}{4} n^{3 / 4} \operatorname{div} \mathbf{V}\right)+\nabla n^{\gamma}=-n \nabla \Phi, \\
\Delta \Phi=\rho-n,
\end{gathered}
$$

where $0<\varepsilon<1$ is a constant.

Set $\rho(\mathbf{x}, t)=\rho(r, t), \mathbf{U}(\mathbf{x}, t)=u(r, t)(\mathbf{x} / r), n(\mathbf{x}, t)=n(r, t)$, $\mathbf{V}(\mathbf{x}, t)=v(r, t)(\mathbf{x} / r)$, and $\Phi(\mathbf{x}, t)=\Phi(r, t)$, and rewrite $(15)$ in the form

$$
\begin{gathered}
\rho_{t}+(\rho u)_{r}+\frac{2 \rho u}{r}=0 \\
(\rho u)_{t}+\left(\rho u^{2}+\rho^{\gamma}\right)_{r}+\frac{2 \rho u^{2}}{r}+\frac{2 u}{r}\left(\rho+\varepsilon \rho^{3 / 4}\right)_{r} \\
=\left(\left(\rho+\frac{3 \varepsilon}{4} \rho^{3 / 4}\right)\left(u_{r}+\frac{2 u}{r}\right)\right)_{r}+\frac{\rho}{r^{2}} \int_{\varepsilon}^{r}(\rho-n) s^{2} \mathrm{~d} s \\
n_{t}+(n v)_{r}+\frac{2 n v}{r}=0, \\
(n v)_{t}+\left(n v^{2}+n^{\gamma}\right)_{r}+\frac{2 n v^{2}}{r}+\frac{2 v}{r}\left(n+\varepsilon n^{3 / 4}\right)_{r} \\
=\left(\left(n+\frac{3 \varepsilon}{4} n^{3 / 4}\right)\left(v_{r}+\frac{2 v}{r}\right)\right)_{r}-\frac{n}{r^{2}} \int_{\varepsilon}^{r}(\rho-n) s^{2} \mathrm{~d} s
\end{gathered}
$$

for $r>0$. We will first construct the smooth solution of (16) in the truncated region $0<\varepsilon<r<1$ with the initial condition

$$
\begin{aligned}
& (\rho, \rho u)(r, 0)=\left(\rho_{0}+\varepsilon, m_{10}\right), \\
& (n, n v)(r, 0)=\left(n_{0}+\varepsilon, m_{20}\right),
\end{aligned}
$$

and the boundary condition

$$
\begin{aligned}
& \left.u(r, t)\right|_{r=\varepsilon}=\left.u(r, t)\right|_{r=1}=0, \\
& \left.v(r, t)\right|_{r=\varepsilon}=\left.v(r, t)\right|_{r=1}=0 .
\end{aligned}
$$

For the approximate solution which will have lower bound of the density (see Lemma 8), the boundary condition of (18) is equivalent to $\left.\rho u(r, t)\right|_{r=\varepsilon}=\left.\rho u(r, t)\right|_{r=1}=0$, $\left.n v(r, t)\right|_{r=\varepsilon}=\left.n v(r, t)\right|_{r=1}=0$.

We assume that the initial data is smooth and satisfies (9) with constants independent of $\varepsilon$.

In the following, we will state the energy and entropy estimates for approximate solution $\left(\rho, n, u, v, \Phi_{r}\right)$. 
First, making use of similar arguments as in [16] with modifications, we can establish the following Lemma 3, of which we omit the details.

Lemma 3. Let $\left(\rho, n, u, v, \Phi_{r}\right)$ be smooth solution of (16) defined on $[\varepsilon, 1] \times[0, T]$ with boundary conditions (18) such that $\rho>0, n>0$. Then there exists a constant $C$, independent of $\varepsilon$, such that

$$
\begin{gathered}
\int_{\varepsilon}^{1}(\rho(r, t)+n(r, t)) r^{2} \mathrm{~d} r \leq C \\
\int_{\varepsilon}^{1}\left(\frac{1}{2} \rho u^{2}+\frac{1}{2} n v^{2}+\frac{1}{\gamma-1} \rho^{\gamma}+\frac{1}{\gamma-1} n^{\gamma}+\frac{1}{2} \Phi_{r}^{2}\right) r^{2} \mathrm{~d} r \\
+\int_{0}^{T} \int_{\varepsilon}^{1}\left(\left(\rho+\frac{\varepsilon}{4} \rho^{3 / 4}\right)\left(r^{2} u_{r}^{2}+u^{2}\right)\right. \\
\left.+\left(n+\frac{\varepsilon}{4} n^{3 / 4}\right)\left(r^{2} v_{r}^{2}+v^{2}\right)\right) \mathrm{d} r \mathrm{~d} t \leq C \\
\int_{\varepsilon}^{1}\left(\frac{1}{2} \rho\left|u+(\log \rho)_{r}+\frac{3 \varepsilon}{4} \rho^{-5 / 4} \rho_{r}\right|^{2}\right. \\
\left.+\frac{1}{2} n\left|v+(\log n)_{r}+\frac{3 \varepsilon}{4} n^{-5 / 4} n_{r}\right|^{2}\right) r^{2} \mathrm{~d} r \\
+\int_{0}^{T} \int_{\varepsilon}^{1}\left(\left(\gamma \rho^{\gamma-2}+\frac{3 \varepsilon}{4} \gamma \rho^{\gamma-9 / 4}\right) \rho_{r}^{2}\right. \\
+\int_{0}^{T} \int_{\varepsilon}^{1}\left((\rho-n)^{2}+\varepsilon\left(\rho^{3 / 4}-n^{3 / 4}\right)(\rho-n)\right) r^{2} \mathrm{~d} r \mathrm{~d} t \\
\left.+\left(\gamma n^{\gamma-2}+\frac{3 \varepsilon}{4} \gamma n^{\gamma-9 / 4}\right) n_{r}^{2}\right) r^{2} \mathrm{~d} r \mathrm{~d} t
\end{gathered}
$$

$\leq C$.

Lemma 4. Given $\varepsilon>0$, there is an absolute constant $C$, which is independent of $\varepsilon$, such that

$$
0 \leq \rho(r, t) \leq \frac{C}{\varepsilon^{2}}, \quad 0 \leq n(r, t) \leq \frac{C}{\varepsilon^{2}},
$$

for $\varepsilon \leq r \leq 1$ and $t \geq 0$.

Proof. Define characteristic line: $\mathrm{d} r(t) / \mathrm{d} t=u(r(t), t)$. Then, along the particle path, $(16)_{1}$ can be solved to obtain

$$
\rho(r(t), t) r^{2}=\rho_{0}(r(0)) r(0)^{2} e^{-\int_{0}^{t} \partial_{r} u(r(s), s) \mathrm{d} s},
$$

which implies that $\rho \geq 0$ provided that $\rho_{0} \geq 0$.

It follows from (20) and (21) that

$$
\int_{\varepsilon}^{1} \frac{\rho_{r}^{2}}{\rho} r^{2} \mathrm{~d} r \leq C
$$

for some absolute constant $C$ independent of $\varepsilon$.
Then, it follows from (19) and (24) that

$$
\begin{aligned}
\rho(r, t) & \leq \int_{\varepsilon}^{1} \rho(r, t) \mathrm{d} r+\int_{\varepsilon}^{1}\left|\partial_{r} \rho(r, t)\right| \mathrm{d} r \\
& \leq \frac{1}{\varepsilon^{2}} \int_{\varepsilon}^{1} \rho(r, t) r^{2} \mathrm{~d} r+\frac{1}{\varepsilon^{2}}\left(\int_{\varepsilon}^{1} \rho r^{2} \mathrm{~d} r+\int_{\varepsilon}^{1} \frac{\rho_{r}^{2}}{\rho} r^{2} \mathrm{~d} r\right) \\
& \leq \frac{C}{\varepsilon^{2}}
\end{aligned}
$$

for $\varepsilon \leq r \leq 1$ and $t \geq 0$.

Similarly, we also have

$$
0 \leq n(r, t) \leq \frac{C}{\varepsilon^{2}}
$$

The proof of the lemma is finished.

To derive a priori estimates about the velocity of the approximate solution, the crucial step is to obtain lower bounds of the density. For this purpose and for simplicity, we solve the IBVP (16) in Lagrangian coordinates. Since the process is the same, we just deal with $(16)_{1}-(16)_{2}$.

Let $\varepsilon>0$ be fixed and define

$$
x(r, t)=\int_{\varepsilon}^{r} \rho r^{2} \mathrm{~d} r, \quad \tau=t .
$$

Without loss of generality, we set $\int_{\mathcal{E}}^{1} \rho r^{2} \mathrm{~d} r=1$. Then,

$$
\frac{\partial x}{\partial r}=\rho r^{2}, \quad \frac{\partial x}{\partial t}=-\rho r^{2} u, \quad \frac{\partial \tau}{\partial r}=0, \quad \frac{\partial \tau}{\partial t}=1,
$$

and $(16)_{1}-(16)_{2}$ becomes

$$
\begin{aligned}
\rho_{\tau} & +\rho^{2}\left(r^{2} u\right)_{x}=0, \\
r^{-2} u_{\tau}+\left(\rho^{\gamma}\right)_{x}= & \left(\left(\rho^{2}+\frac{3 \varepsilon}{4} \rho^{7 / 4}\right)\left(r^{2} u\right)_{x}\right)_{x} \\
& -\frac{2 u}{r}\left(\rho+\varepsilon \rho^{3 / 4}\right)_{x}+\rho \Phi_{x},
\end{aligned}
$$

for $\tau>0$ and $0 \leq x \leq 1$.

The corresponding initial data is

$$
(\rho, \rho u)(x, 0)=\left(\rho_{0}+\varepsilon, m_{10}\right),
$$

and the boundary condition is

$$
u(0, \tau)=0, \quad u(1, \tau)=0
$$

For this system, the following a priori estimates hold. 
Abstract and Applied Analysis

5

Lemma 5. For all $\tau \in[0, T]$, it holds that

$$
\begin{gathered}
\int_{0}^{1}\left(\frac{1}{2} u^{2}+\frac{1}{\gamma-1} \rho^{\gamma-1}\right) \mathrm{d} x+\int_{0}^{\tau} \int_{0}^{1}\left(\frac{2 u^{2}}{r^{2}}+\rho^{2} r^{4} u_{x}^{2}\right) \mathrm{d} x \mathrm{~d} s \\
+\int_{0}^{\tau} \int_{0}^{1}\left(\frac{\varepsilon}{2} \frac{u^{2}}{\rho^{1 / 4} r^{2}}+\frac{\varepsilon}{4} \rho^{7 / 4} r^{4} u_{x}^{2}\right) \mathrm{d} x \mathrm{~d} s \\
\leq \int_{0}^{1}\left(\frac{1}{2} u_{0}^{2}+\frac{1}{\gamma-1} \rho_{0}^{\gamma-1}\right) \mathrm{d} x+C(\varepsilon, T),
\end{gathered}
$$$$
0 \leq \rho(x, \tau) \leq C(\varepsilon, T),
$$$$
\varepsilon \leq r(x, \tau) \leq 1,
$$

$$
\begin{aligned}
\int_{0}^{1} u^{4} \mathrm{~d} x+\int_{0}^{\tau} \int_{0}^{1}\left(\frac{4 u^{2}}{r^{2}}+6 \rho^{2} r^{4} u^{4} u_{x}^{2}\right. \\
\left.+\frac{2 \varepsilon u^{4}}{\rho^{1 / 4} r^{2}}+\varepsilon \rho^{7 / 4} r^{4} u^{2} u_{x}^{2}\right) \mathrm{d} x \mathrm{~d} s
\end{aligned}
$$

$$
\leq \int_{0}^{1} u_{0}^{4} \mathrm{~d} x+C(\varepsilon, T) .
$$

Proof. Multiplying (29) 2 by $r^{2} u$, using (29) ${ }_{1}$ and integration by parts, we can get

$$
\begin{aligned}
& \frac{\mathrm{d}}{\mathrm{d} \tau} \int_{0}^{1}\left(\frac{1}{2} u^{2}+\frac{1}{\gamma-1} \rho^{\gamma-1}\right) \mathrm{d} x \\
& \quad+\int_{0}^{1}\left(\rho^{2}+\frac{3 \varepsilon}{4} \rho^{7 / 4}\right)\left(\left(r^{2} u\right)_{x}\right)^{2} \mathrm{~d} x \\
& =\int_{0}^{1}\left(\rho+\varepsilon \rho^{3 / 4}\right)\left(2 r u^{2}\right)_{x} \mathrm{~d} x+\int_{0}^{1} \rho r^{2} u \Phi_{x} \mathrm{~d} x \\
& =4 \int_{0}^{1}\left(\rho+\varepsilon \rho^{3 / 4}\right) u u_{x} r \mathrm{~d} x+2 \int_{0}^{1}\left(1+\varepsilon \rho^{-1 / 4}\right) \frac{u^{2}}{r^{2}} \mathrm{~d} x \\
& \quad+\int_{0}^{1} \rho r^{2} u \Phi_{x} \mathrm{~d} x .
\end{aligned}
$$

Since

$$
\left(\left(r^{2} u\right)_{x}\right)^{2}=\left(\frac{2 u}{r \rho}+r^{2} u_{x}\right)^{2}=\frac{4 u^{2}}{\rho^{2} r^{2}}+\frac{4 r u u_{x}}{\rho}+r^{4} u_{x}^{2},
$$

then from (36), we get

$$
\begin{gathered}
\frac{\mathrm{d}}{\mathrm{d} \tau} \int_{0}^{1}\left(\frac{1}{2} u^{2}+\frac{1}{\gamma-1} \rho^{\gamma-1}\right) \mathrm{d} x+\int_{0}^{1}\left(\frac{2 u^{2}}{r^{2}}+\rho^{2} u_{x}^{2} r^{4}\right) \mathrm{d} x \\
+\int_{0}^{1}\left(\frac{\varepsilon u^{2}}{\rho^{1 / 4} r^{2}}+\frac{3 \varepsilon}{4} \rho^{7 / 4} u_{x}^{2} r^{4}\right) \mathrm{d} x \\
=\varepsilon \int_{0}^{1} \rho^{3 / 4} u u_{x} r \mathrm{~d} x+\int_{0}^{1} \rho r^{2} u \Phi_{x} \mathrm{~d} x
\end{gathered}
$$

$$
\begin{aligned}
\leq & \frac{1}{2} \int_{0}^{1} \frac{\varepsilon u^{2}}{\rho^{1 / 4} r^{2}} \mathrm{~d} x+\frac{1}{2} \int_{0}^{1} \varepsilon \rho^{7 / 4} u_{x}^{2} r^{4} \mathrm{~d} x \\
& +\left(\int_{0}^{1} \rho r^{4} \Phi_{x}^{2} \mathrm{~d} x\right)^{1 / 2}\left(\int_{0}^{1} \rho u^{2} \mathrm{~d} x\right)^{1 / 2} \\
\leq & \frac{1}{2} \int_{0}^{1} \frac{\varepsilon u^{2}}{\rho^{1 / 4} r^{2}} \mathrm{~d} x+\frac{1}{2} \int_{0}^{1} \varepsilon \rho^{7 / 4} u_{x}^{2} r^{4} \mathrm{~d} x+C(\varepsilon) .
\end{aligned}
$$

Thus (32) holds.

Next, (33) follows from Lemma 4 and (34) holds trivially.

Now, we prove (35). In fact, multiplying $(29)_{2}$ by $r^{2} u^{3}$, using $(29)_{1}$ and integration by parts, we get

$$
\begin{aligned}
\frac{1}{4} \frac{\mathrm{d}}{\mathrm{d} \tau} \int_{0}^{1} u^{4} \mathrm{~d} x & +\int_{0}^{1}\left(\rho^{2}+\frac{3 \varepsilon}{4} \rho^{7 / 4}\right)\left(\left(r^{2} u\right)_{x}\right)^{2} u^{2} \mathrm{~d} x \\
& +2 \int_{0}^{1}\left(\rho^{2}+\frac{3 \varepsilon}{4} \rho^{7 / 4}\right) u^{2} u_{x}^{2} r^{4} \mathrm{~d} x \\
= & -4 \int_{0}^{1}\left(\rho+\frac{3 \varepsilon}{4} \rho^{3 / 4}\right) u^{3} u_{x} r \mathrm{~d} x+\int_{0}^{1} \rho^{\gamma}\left(u^{3} r^{2}\right)_{x} \mathrm{~d} x \\
& +\int_{0}^{1}\left(\rho+\varepsilon \rho^{3 / 4}\right)\left(2 u^{4} r\right)_{x} \mathrm{~d} x+\int_{0}^{1} \rho \Phi_{x} r^{2} u^{3} \mathrm{~d} x .
\end{aligned}
$$

Thus

$$
\begin{aligned}
\frac{1}{4} \frac{\mathrm{d}}{\mathrm{d} \tau} \int_{0}^{1} u^{4} \mathrm{~d} x & +\int_{0}^{1}\left(\frac{2 u^{4}}{r^{2}}+3 \rho^{2} u^{2} u_{x}^{2} r^{4}\right) \mathrm{d} x \\
& +\int_{0}^{1}\left(\frac{\varepsilon u^{4}}{\rho^{1 / 4} r^{2}}+\frac{9 \varepsilon}{4} \rho^{7 / 4} u^{2} u_{x}^{2} r^{4}\right) \mathrm{d} x \\
= & 2 \int_{0}^{1} \varepsilon \rho^{3 / 4} u^{3} u_{x} r \mathrm{~d} x+\int_{0}^{1}\left(2 \rho^{\gamma-1} \frac{u^{3}}{r}+3 \rho^{\gamma} u^{2} u_{x} r^{2}\right) \mathrm{d} x \\
& +\int_{0}^{1} \rho \Phi_{x} r^{2} u^{3} \mathrm{~d} x .
\end{aligned}
$$

Using Hölder inequality, Young's inequality, and Lemma 4, we estimate the right hand side of (40) as follows:

$$
\begin{aligned}
2 \int_{0}^{1} \varepsilon \rho^{3 / 4} u^{3} u_{x} r \mathrm{~d} x \leq & \frac{1}{2} \int_{0}^{1} \frac{\varepsilon u^{4}}{\rho^{1 / 4} r^{2}} \mathrm{~d} x+2 \int_{0}^{1} \varepsilon \rho^{7 / 4} u^{2} u_{x}^{2} r^{4} \mathrm{~d} x \\
2 \int_{0}^{1} \rho^{\gamma-1} \frac{u^{3}}{r} \mathrm{~d} x & \leq 2\left(\int_{0}^{1} \rho^{4(\gamma-1)} r^{2} \mathrm{~d} x\right)^{1 / 4}\left(\int_{0}^{1} \frac{u^{4}}{r^{2}} \mathrm{~d} x\right)^{3 / 4} \\
\leq & \frac{1}{3} \int_{0}^{1} \frac{u^{4}}{r^{2}} \mathrm{~d} x+C \\
3 \int_{0}^{1} \rho^{\gamma} u^{2} u_{x} r^{2} \mathrm{~d} x & \leq 3\left(\int_{0}^{1} \rho^{2 \gamma-2} u^{2} \mathrm{~d} x\right)^{1 / 2} \\
& \times\left(\int_{0}^{1} \rho^{2} u^{2} u_{x}^{2} r^{4} \mathrm{~d} x\right)^{1 / 2}
\end{aligned}
$$


6

Abstract and Applied Analysis

$\leq \frac{3}{2} \int_{0}^{1} \rho^{2 \gamma-2} u^{2} \mathrm{~d} x+\frac{3}{2} \int_{0}^{1} \rho^{2} u^{2} u_{x}^{2} r^{4} \mathrm{~d} x$

$\leq \frac{3}{2} \int_{0}^{1} \rho^{2} u^{2} u_{x}^{2} r^{4} \mathrm{~d} x+\frac{1}{3} \int_{0}^{1} \frac{u^{4}}{r^{2}} \mathrm{~d} x+C$,

$$
\begin{aligned}
\int_{0}^{1} \rho \Phi_{x} r^{2} u^{3} \mathrm{~d} x & =\int_{\varepsilon}^{1} \rho r^{2} u^{3} \Phi_{r} \mathrm{~d} r \\
& =\int_{\varepsilon}^{1} \rho u^{3}\left(\int_{\varepsilon}^{r}(\rho-n) s^{2} \mathrm{~d} s\right) \mathrm{d} r \\
& \leq C(\varepsilon, T) \int_{\varepsilon}^{1} \rho u^{3} \mathrm{~d} r \\
& =C(\varepsilon, T) \int_{0}^{1} \frac{u^{3}}{r^{2}} \mathrm{~d} x \\
& \leq \frac{1}{3} \int_{0}^{1} \frac{u^{4}}{r^{2}} \mathrm{~d} x+C(\varepsilon, T) \int_{0}^{1} \frac{u^{2}}{r^{2}} \mathrm{~d} x .
\end{aligned}
$$

Putting the above estimates into (40) and using (32), one gets

$$
\begin{aligned}
& \int_{0}^{1} u^{4} \mathrm{~d} x \\
& \quad+\int_{0}^{\tau} \int_{0}^{1}\left(\frac{4 u^{4}}{r^{2}}+6 \rho^{2} u^{2} u_{x}^{2} r^{4}+\frac{2 \varepsilon u^{4}}{\rho^{1 / 4} r^{2}}+\varepsilon \rho^{7 / 4} u^{2} u_{x}^{2} r^{4}\right) \mathrm{d} x \mathrm{~d} s \\
& \leq \int_{0}^{1} u_{0}^{4} \mathrm{~d} x+C(\varepsilon, T) .
\end{aligned}
$$

This proves (35).

Remark 6. Consider

$$
\int_{0}^{1} u_{0}^{4} \mathrm{~d} x=\int_{\varepsilon}^{1} \frac{m_{10}^{4}}{\left(\rho_{0}+\varepsilon\right)^{4}} \rho r^{2} \mathrm{~d} r \leq C(\varepsilon)\left\|\mathbf{m}_{10}\right\|_{L^{4}(\Omega)}^{4} .
$$

Lemma 7. There is a positive constant $C=$ $C\left(\varepsilon, T,\left\|\rho_{0}\right\|_{W^{1,4}(\Omega)},\left\|\mathbf{m}_{10}\right\|_{L^{4}(\Omega)}\right)$ such that

$$
\int_{0}^{1}\left(\left(\rho^{3 / 4}\right)_{x}\right)^{4}(x, \tau) \mathrm{d} x \leq C, \quad \forall \tau \in[0, T] .
$$

Proof. We rewrite $(29)_{1}$ in the form

$$
\left(\rho+\varepsilon \rho^{3 / 4}\right)_{x \tau}=-\left[\left(\rho^{2}+\frac{3 \varepsilon}{4} \rho^{7 / 4}\right)\left(r^{2} u\right)_{x}\right]_{x} .
$$

Then substituting (45) into (29) ${ }_{2}$, one gets

$$
r^{-2} u_{\tau}+\left(\rho^{\gamma}\right)_{x}=-\left(\rho+\varepsilon \rho^{3 / 4}\right)_{x \tau}-\frac{2 u}{r}\left(\rho+\varepsilon \rho^{3 / 4}\right)_{x}+\rho \Phi_{x}
$$

that is,

$$
r^{2}\left(\rho+\varepsilon \rho^{3 / 4}\right)_{x \tau}+2 u r\left(\rho+\varepsilon \rho^{3 / 4}\right)_{x}=-u_{\tau}-r^{2}\left(\rho^{\gamma}\right)_{x}+\rho r^{2} \Phi_{x} .
$$

Since $\partial r / \partial \tau=u$, the above equation can be rewritten as

$$
\left(r^{2}\left(\rho+\varepsilon \rho^{3 / 4}\right)_{x}\right)_{\tau}=-u_{\tau}-r^{2}\left(\rho^{\gamma}\right)_{x}+\rho r^{2} \Phi_{x} .
$$

Integrating it over $[0, \tau]$, one gets

$$
\begin{aligned}
u(x, \tau) & -u_{0}(x)+\int_{0}^{\tau} r^{2}\left(\rho^{\gamma}\right)_{x}(x, s) \mathrm{d} s \\
= & r_{0}^{2}\left(\frac{4}{3} \rho_{0}^{1 / 4}+\varepsilon\right) \partial_{x}\left(\rho_{0}^{3 / 4}\right)-\left(\frac{4}{3} \rho^{1 / 4}+\varepsilon\right) r^{2} \partial_{x}\left(\rho^{3 / 4}\right) \\
& +\int_{0}^{\tau} \rho r^{2} \Phi_{x} \mathrm{~d} s .
\end{aligned}
$$

Multiplying (49) by $\left(r^{2} \partial_{x}\left(\rho^{3 / 4}\right)\right)^{3}$ and integrating over $[0,1]$ with respect to $x$, one gets

$$
\begin{aligned}
\int_{0}^{1}( & \left.\frac{4}{3} \rho^{1 / 4}+\varepsilon\right)\left(r^{2} \partial_{x}\left(\rho^{3 / 4}\right)\right)^{4} \mathrm{~d} x \\
= & \int_{0}^{1} r_{0}^{2}\left(\frac{4}{3} \rho_{0}^{1 / 4}+\varepsilon\right) \partial_{x}\left(\rho_{0}^{3 / 4}\right)\left(r^{2} \partial_{x}\left(\rho^{3 / 4}\right)\right)^{3} \mathrm{~d} x \\
& +\int_{0}^{1}\left(r^{2} \partial_{x}\left(\rho^{3 / 4}\right)\right)^{3} \int_{0}^{\tau} \rho r^{2} \Phi_{x} \mathrm{~d} s \mathrm{~d} x \\
& -\int_{0}^{1}\left\{u-u_{0}+\int_{0}^{\tau} r^{2}\left(\rho^{\gamma}\right)_{x}(x, s) \mathrm{d} s\right\}\left(r^{2} \partial_{x}\left(\rho^{3 / 4}\right)\right)^{3} \mathrm{~d} x \\
\leq & C\left(\int_{0}^{1}\left(r^{2} \partial_{x}\left(\rho^{3 / 4}\right)\right)^{4} \mathrm{~d} x\right)^{3 / 4} \\
\quad \times\left\{\left\|u-u_{0}\right\|_{L^{4}}+\left\|\partial_{x}\left(\rho_{0}^{3 / 4}\right)\right\|_{L^{4}}\right. & \left\{\left(\int_{0}^{\tau}\left\|\rho r^{2} \Phi_{x}\right\|_{L^{4}}^{4} \mathrm{~d} s\right)^{1 / 4}+\left(\int_{0}^{\tau}\left\|\partial_{x} \rho^{\gamma}\right\|_{L^{4}}^{4} \mathrm{~d} s\right)^{1 / 4}\right\},
\end{aligned}
$$

in which

$$
\begin{aligned}
\int_{0}^{\tau}\left\|\rho r^{2} \Phi_{x}\right\|_{L^{4}}^{4} \mathrm{~d} s & =\int_{0}^{\tau} \int_{0}^{1}\left(\rho r^{2} \Phi_{x}\right)^{4} \mathrm{~d} x \mathrm{~d} s \\
& =\int_{0}^{\tau} \int_{\mathcal{\varepsilon}}^{1} \rho r^{2} \Phi_{r}^{4} \mathrm{~d} r \mathrm{~d} s \\
& =\int_{0}^{\tau} \int_{\mathcal{\varepsilon}}^{1} \rho r^{2}\left(\frac{1}{r^{2}} \int_{\varepsilon}^{r}(\rho-n) s^{2} \mathrm{~d} s\right)^{4} \mathrm{~d} r \mathrm{~d} s \\
& \leq C(\varepsilon, T) .
\end{aligned}
$$

Using Lemma 5 and Young's inequality, we deduce from (50) that there is a positive constant $C$, depending on $\left\|\rho_{0}\right\|_{W^{1,4}[0,1]}$, $\left\|u_{0}\right\|_{L^{4}[0,1]}, \mathcal{\varepsilon}$, and $T$, such that

$$
\begin{aligned}
\varepsilon \int_{0}^{1}\left(r^{2} \partial_{x}\left(\rho^{3 / 4}\right)\right)^{4} \mathrm{~d} x \leq & \frac{\varepsilon}{2} \int_{0}^{1}\left(r^{2} \partial_{x}\left(\rho^{3 / 4}\right)\right)^{4} \mathrm{~d} x \\
& +C \int_{0}^{\tau} \int_{0}^{1}\left(\partial_{x} \rho^{\gamma}\right)^{4} \mathrm{~d} x \mathrm{~d} s+C ;
\end{aligned}
$$


that is,

$$
\begin{aligned}
& \int_{0}^{1}\left(\partial_{x}\left(\rho^{3 / 4}\right)\right)^{4} \mathrm{~d} x \\
& \quad \leq C+C \int_{0}^{\tau} \max _{[0,1]}\left(\rho^{4 \gamma-3}\right) \int_{0}^{1}\left(\partial_{x}\left(\rho^{3 / 4}\right)\right)^{4} \mathrm{~d} x \mathrm{~d} s .
\end{aligned}
$$

Applying Gronwall's inequality to (53), we have

$$
\int_{0}^{1}\left(\partial_{x}\left(\rho^{3 / 4}\right)\right)^{4} \mathrm{~d} x \leq C .
$$

This proves (44).

Now we can obtain the lower bound of the density $\rho$.

Lemma 8. There is a positive constant $C=C(\varepsilon, T$, $\left.\left\|\rho_{0}\right\|_{W^{1,4}(\Omega)},\left\|\mathbf{m}_{10}\right\|_{L^{4}(\Omega)}\right)$ such that

$$
\rho \geq C, \quad \forall x \in[0,1], \tau \in[0, T] .
$$

Proof. Set $v(x, \tau)=1 / \rho(x, \tau)$ and $V(\tau)=\max _{[0,1] \times[0, \tau]} v(x, s)$. Equation $(29)_{1}$ can be written as $v_{\tau}=\left(r^{2} u\right)_{x}$, which implies that $\int_{0}^{1} v(x, \tau) \mathrm{d} x=\int_{0}^{1} v(x, 0) \mathrm{d} x \leq C_{0}$. Then it follows from Sobolev's embedding $W^{1,1}([0,1]) \hookrightarrow L^{\infty}([0,1])$ that, for any $0<\beta<1$,

$$
\begin{aligned}
v^{\beta}(x, \tau) \leq & \int_{0}^{1} v^{\beta}(x, \tau) \mathrm{d} x+\int_{0}^{1}\left|\partial_{x} v^{\beta}\right| \mathrm{d} x \\
\leq & \left(\int_{0}^{1} v \mathrm{~d} x\right)^{\beta}+\beta \int_{0}^{1} v^{\beta+3 / 4}\left|\frac{\rho_{x}}{\rho^{1 / 4}}\right| \mathrm{d} x \\
\leq & C+C \beta\left(\int_{0}^{1}\left(v^{\beta+3 / 4}\right)^{4 / 3} \mathrm{~d} x\right)^{3 / 4} \\
& \times\left(\int_{0}^{1}\left(\left(\rho^{3 / 4}\right)_{x}\right)^{4} \mathrm{~d} x\right)^{1 / 4} \\
\leq & C+C \beta V^{\beta}\left(\int_{0}^{1} v \mathrm{~d} x\right)^{3 / 4}\left(\int_{0}^{1}\left(\left(\rho^{3 / 4}\right)_{x}\right)^{4} d x\right)^{1 / 4} \\
\leq & C+C \beta V^{\beta} .
\end{aligned}
$$

Choosing $\beta>0$ small enough, which may depend on $\varepsilon$ and $T$, we obtain

$$
V(\tau) \leq C,
$$

where $C=C\left(\varepsilon, T,\left\|\rho_{0}\right\|_{W^{1,4}(\Omega)},\left\|\mathbf{m}_{10}\right\|_{L^{4}(\Omega)}\right)$. The proof of the lemma is completed.

\section{Proof of Theorem 2}

Proof. With the estimates obtained in Section 3, we can apply the method in [16] and references therein with modifications to prove the existence of weak solution to the IBVP (2). The details are omitted.

\section{Conflict of Interests}

The authors declare that there is no conflict of interests regarding the publication of this paper.

\section{Authors' Contribution}

All authors contributed to each part of this work equally.

\section{Acknowledgments}

The authors are grateful to Professor Hai-Liang Li for his helpful discussions and suggestions about the problem. The research of Jian Liu is supported by NNSFC no. 11326140 and the Doctoral Starting up Foundation of Quzhou University no. BSYJ201314 and no. XNZQN201313.

\section{References}

[1] D. Donatelli, "Local and global existence for the coupled NavierStokes-Poisson problem," Quarterly of Applied Mathematics, vol. 61, no. 2, pp. 345-361, 2003.

[2] Y. Zhang and Z. Tan, "On the existence of solutions to the Navier-Stokes-Poisson equations of a two-dimensional compressible flow," Mathematical Methods in the Applied Sciences, vol. 30, no. 3, pp. 305-329, 2007.

[3] D. Donatelli and P. Marcati, "A quasineutral type limit for the Navier-Stokes-Poisson system with large data," Nonlinearity, vol. 21, no. 1, pp. 135-148, 2008.

[4] Q. Ju, F. Li, and H. Li, "The quasineutral limit of compressible Navier-Stokes-Poisson system with heat conductivity and general initial data," Journal of Differential Equations, vol. 247, no. 1, pp. 203-224, 2009.

[5] S. Wang and S. Jiang, "The convergence of the Navier-StokesPoisson system to the incompressible Euler equations," Communications in Partial Differential Equations, vol. 31, no. 4-6, pp. 571-591, 2006.

[6] B. Ducomet and A. Zlotnik, "Stabilization and stability for the spherically symmetric Navier-Stokes-Poisson system," Applied Mathematics Letters, vol. 18, no. 10, pp. 1190-1198, 2005.

[7] R. X. Lian and M. Li, "Stability of weak solutions for the compressible Navier-Stokes-Poisson equations," Acta Mathematicae Applicatae Sinica, vol. 28, no. 3, pp. 597-606, 2012.

[8] C. Hao and H.-L. Li, "Global existence for compressible NavierStokes-Poisson equations in three and higher dimensions," Journal of Differential Equations, vol. 246, no. 12, pp. 4791-4812, 2009.

[9] H.-L. Li, A. Matsumura, and G. Zhang, "Optimal decay rate of the compressible Navier-Stokes-Poisson system in $\mathrm{R}^{3}$," Archive for Rational Mechanics and Analysis, vol. 196, no. 2, pp. 681-713, 2010.

[10] H.-L. Li, T. Yang, and C. Zou, "Time asymptotic behavior of the bipolar Navier-Stokes-Poisson system," Acta Mathematica Scientia B, vol. 29, no. 6, pp. 1721-1736, 2009.

[11] L. Hsiao, H.-L. Li, T. Yang, and C. Zou, "Compressible nonisentropic bipolar navier-stokes-poisson system in $\mathbb{R}^{3}$," Acta Mathematica Scientia, vol. 31, no. 6, pp. 2169-2194, 2011.

[12] C. Zou, "Large time behaviors of the isentropic bipolar compressible Navier-Stokes-Poisson system," Acta Mathematica Scientia B: English Edition, vol. 31, no. 5, pp. 1725-1740, 2011. 
[13] J. Liu and R. Lian, "Existence of global solutions to free boundary value problems for bipolar Navier-Stokes-Possion systems," Electronic Journal of Differential Equations, vol. 2013, article 200, 2013.

[14] J. Liu, R. X. Lian, and M. F. Qian, "Global existence of solution to Bipolar Navier-Stokes-Poisson system," In press.

[15] Y. Q. Lin, C. C. Hao, and H. -L. Li, "Global well-posedness of compressible bipolar Navier-Stokes-Poisson equations," Acta Mathematica Sinica, vol. 28, no. 5, pp. 925-940, 2012.

[16] Z.-H. Guo, Q.-S. Jiu, and Z.-P. Xin, "Spherically symmetric isentropic compressible flows with density-dependent viscosity coefficients," SIAM Journal on Mathematical Analysis, vol. 39, no. 5, pp. 1402-1427, 2008. 


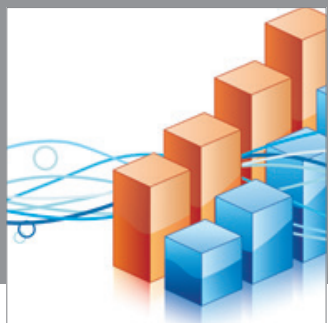

Advances in

Operations Research

mansans

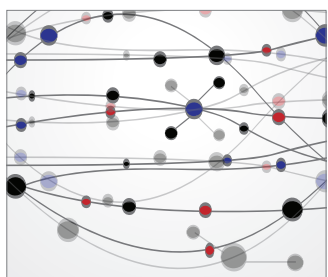

The Scientific World Journal
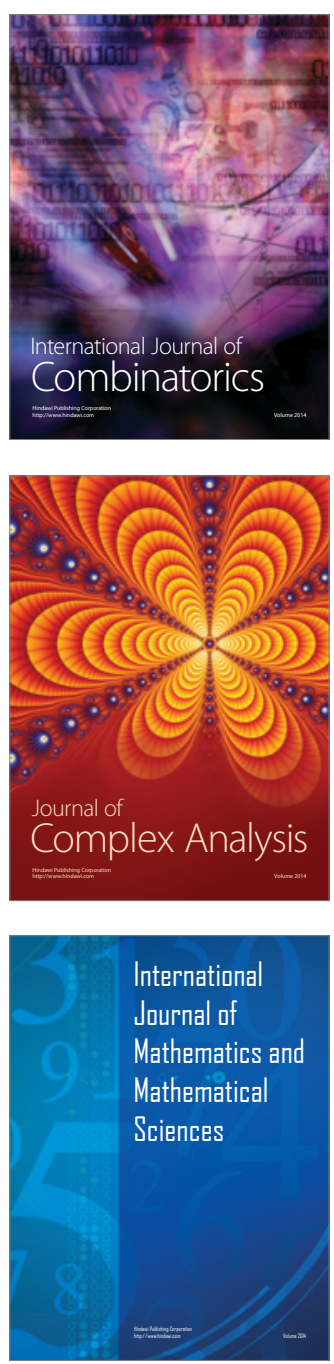
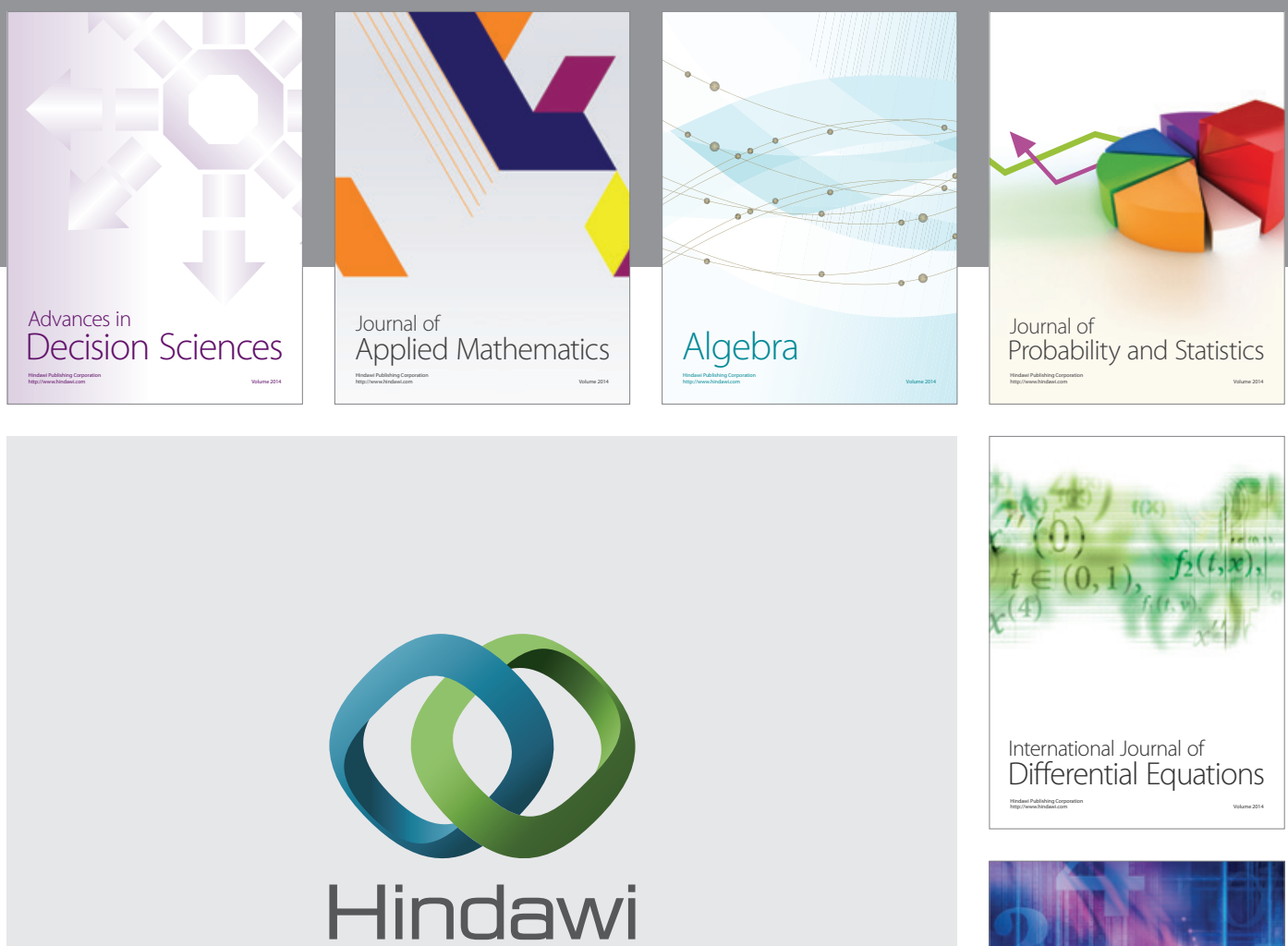

Submit your manuscripts at http://www.hindawi.com
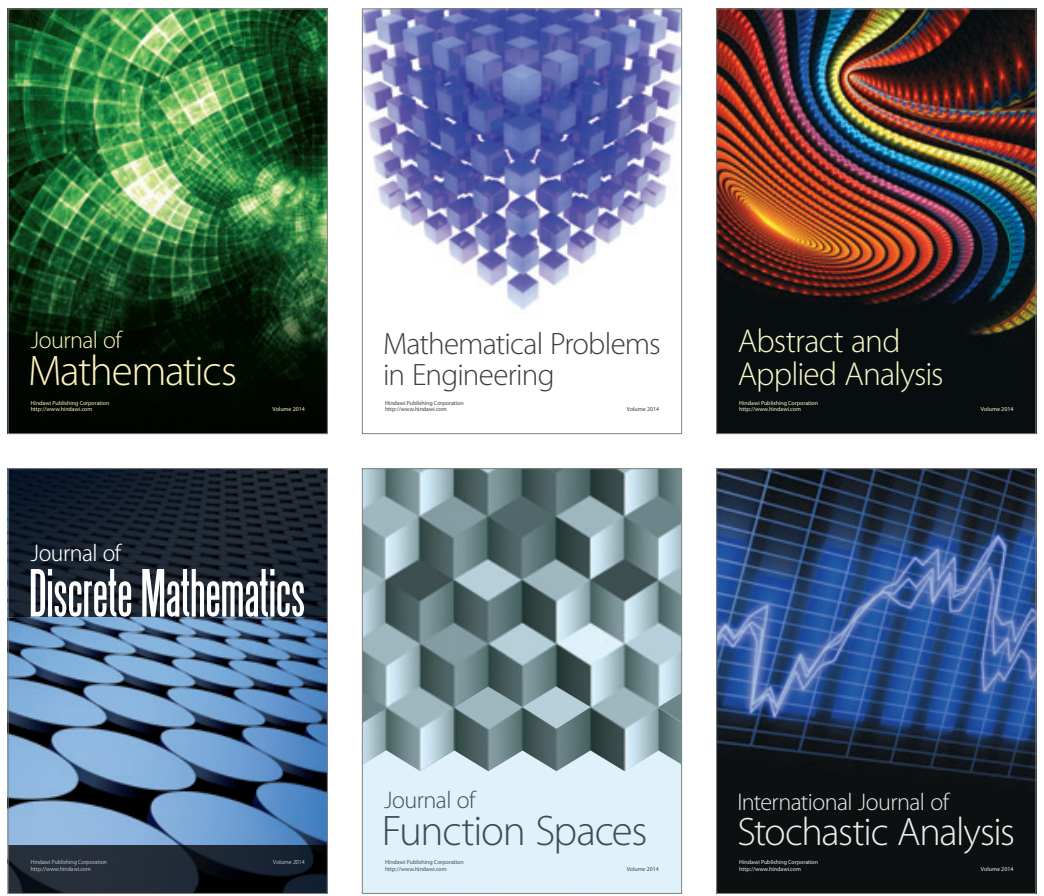

Journal of

Function Spaces

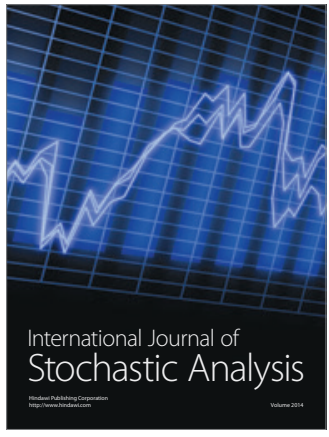

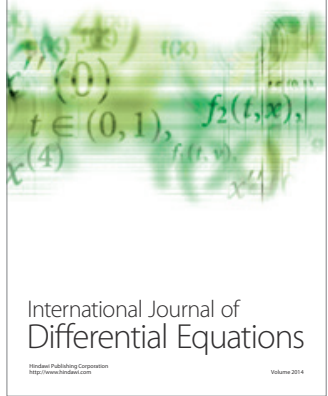
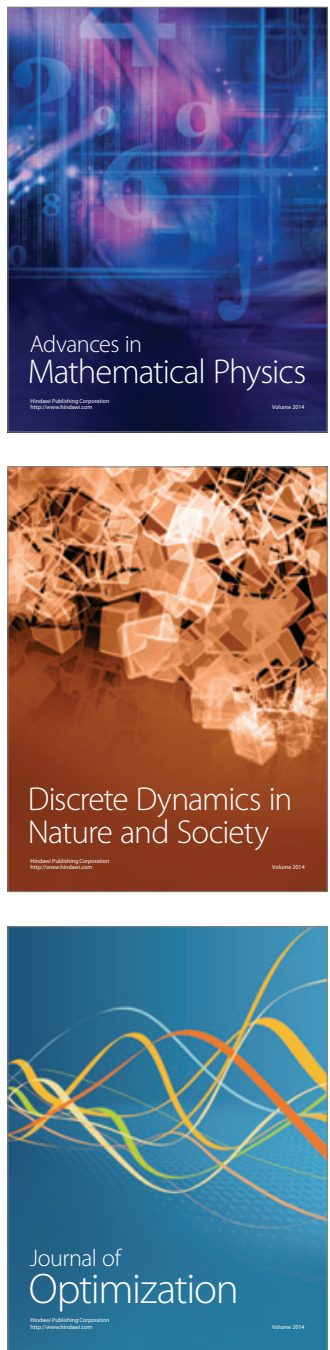